Pacific Journal of Mathematics

WASTES ON BOOLEAN ALCEBBRA 


\section{MEASURES ON BOOLEAN ALGEBRAS}

\section{J. L. KELLEY}

This paper is concerned with the general problem of the existence of measures on Boolean algebras. A measure on a Boolean algebra $\mathscr{A}$ is a finitely additive, non-negative function on $\mathscr{A}$ which assumes the value one at the unit element of the algebra $\mathscr{A}$. It is known that measures on Boolean algebras always exist, and in some profusion (see, for example, [2]). We are concerned primarily with the existence of measures which are strictly positive; that is measures which vanish only at the zero element of the algebra. Not all Boolean algebras possess strictly positive measures, and workable necessary and sufficient conditions for the existence of a strictly positive measure have not been given. We shall give such conditions. Our results seem to represent definite progress on the general problem, although the relationship between our conditions and various conjectures is not clear. In particular, I do not know whether there is necessarily a strictly positive measure on an algebra $\mathscr{A}$ which satisfies the condition: $\mathscr{A}-\{0\}$ is the union of a countable family $\left\{\mathscr{A}_{n}\right\}$, such that each disjoint subclass of the class $\mathscr{A} /$ contains at most $n$ members. Tarski has conjectured that this is the case.

In the first section we define, combinatorially, for each subset $\mathscr{B}$ of a Boolean algebra $\mathscr{A}$ a number, $I(\mathscr{B})$, called the intersection number of $\mathscr{B}$. It is then showed that there is a strictly positive measure on $\mathscr{A}$ if and only if $\mathscr{A}-\{0\}$ is the union of a countable number of sets, each of which has positive intersection number. The intersection number is also evaluated precisely in terms of measures on $\mathscr{A} ; I(\mathscr{B})$ is the maximum, for all measures $m$ on $\mathscr{A}$, of inf $\{m(B): B \in \mathscr{B}\}$. A dualized formulation of these results in terms of coverings is obtained.

The second section is concerned with the existence of countably additive measures. Necessary and sufficient conditions for the existence of such measures have been given by Maharam [3], but these conditions are not entirely satisfactory. The contribution to the problem made here is simply this: an algebra supports a countably additive strictly positive measure if and only if it has a strictly positive measure and is weakly countably distributive. (See the second section for definitions). The condition of weak countable distributivity appears thus as a very natural requirement which enables one to derive countably additive measures from finitely additive ones; the fundamental difficulties lie in the finitely additive case.

It has been shown that some of the natural conjectures on the

Received February 2, 1959. This investigation was made during the tenure of a Fulbright Research Professorship at Cambridge University. 
existence of measures, or of countably additive measures, imply the Souslin hypothesis (cf. [2] and [3]). In the third section of this paper some results of this sort are obtained. Assuming the falsehood of the Souslin hypothesis, a linear continuum is constructed that has appallingly pathological properties. A condition in terms of Boolean algebras which is equivalent to the Souslin hypothesis is given. The last section deals with measures which dominate, or are dominated by, a non-negative function having certain convexity properties.

We shall assume, unless specifically stated otherwise, that the Boolean algebra $\mathscr{A}$ is the algebra of all open and closed subsets of a compact totally disconnected Hausdorff space $X$; the Boolean operations of (finite) join and meet are then simply union and intersection, and $X$ is the unit of the algebra. This assumption is justified since, via the classical Stone theorem, every Boolean algebra can be isomorphically represented in this fashion.

Intersection numbers and covering numbers. Let $\mathscr{B}$ be an arbitrary non-void subclass of a Boolean algebra $\mathscr{A}$. For each finite sequence $S=\left\langle S_{1}, \cdots, S_{n}\right\rangle$ of (not necessarily distinct) members of $\mathscr{B}$ denote by $n(S)$ the number $n$ of terms in the sequence and let $i(S)$ be the maximum number of members with non-void intersection. If $K_{i}$ is the characteristic function of $S_{i}$ then $i(S)=\sup \left\{\sum\left\{K_{i}(x) ; i=1, \cdots n\right\}\right.$ : $x \in X\}$. The intersection number $I(\mathscr{B})$ is defined to be inf $\{i(S) / n(S)$; $S$ a finite sequence in $\mathscr{B}\}$.

1. Proposition. If $m$ is a measure on $\mathscr{A}$ and $\mathscr{B}$ is a non-void subclass of $\mathscr{A}$ then inf $\{m(B): \in \mathscr{B}\} \leq I(\mathscr{B})$, where $I(\mathscr{B})$ is the intersection number of $\mathscr{B}$.

Proof. Let $r=\inf \{m(B): B \in \mathscr{B}\}$, and let $K_{i}$ be the charcteristic function of $S_{i}$ where $S=\left\langle S_{1}, \cdots, S_{n}\right\rangle$ is a finite sequence in $\mathscr{B}$. Then $\int \sum\left\{K_{i}: i=1, \cdots, n\right\} d m=\sum\left\{m\left(S_{i}\right): i=1, \cdots, n\right\} \geq r n$, and therefore $\sum\left\{K_{i}(x): i=1, \cdots, n\right\} \geq r n$ for some $x$ in $X$. Hence the maximum number $i(S)$ of elements of $S$ which intersect is at least $r n$, and $i(S) / n(S) \geq r$. Taking the infimum for all $S$, we have $I(\mathscr{B}) \geq r$.

The preceding proposition can be restated: $I(\mathscr{B}) \geq \sup$ inf $\{m(B)$ : $B \in \mathscr{B}\}$, where the supremum is taken over all measures $m$ on $\mathscr{A}$. The principal result of this section implies that equality holds here and the supremum is assumed.

2. THEOREM. If $\mathscr{B}$ is a non-void subclass of a Boolean algebra $\mathscr{A}$ then there is a measure $m$ on $\mathscr{A}$ such that $\inf \{m(B): B \in \mathscr{B}\}=$ $I(\mathscr{B})$, where $I(\mathscr{B})$ is the intersection number of $\mathscr{B}$.

Proof. Let $C$ be the class of all continuous real valued functions on $X$, with the usual supremum norm, let $F$ be the class of characteristic 
functions of members of $\mathscr{B}$, and let $G$ be the convex hull of $F$. We assert that if $f \in G$ then $\|f\| \geq I(\mathscr{B})$. For suppose that $\left\|\sum\left\{t_{i} K_{i}: i=1, \cdots, q\right\}\right\|=r$, where $K_{i}$ is the characteristic function of a member $S_{i}$ of $\mathscr{B}, 0<t_{i} \leq 1$ for each $i$, and $\sum\left\{t_{i}: i=1, \cdots, q\right\}=1$. For arbitrary $e>0$ we then have $\left\|(1 / n) \sum\left\{p_{i} K: i=1, \cdots, q\right\}\right\|<r$ $+e$ for suitably chosen positive integers $p_{1}, \cdots, p_{q}$ with $\sum\left\{p_{i}: i=1\right.$, $\cdots, q\}=n$. Upon considering the sequence in $\mathscr{B}$ obtained by counting each $S_{i}$ the integer $p_{i}$ times, we see that at least $n I(\mathscr{B})$ of the members of that sequence intersect, whence $I(\mathscr{B}) \leq \|(1 / n) \sum\left\{p_{i} K_{i}: i=1\right.$, $\cdots, q\} \|<r+e$. Consequently $I(\mathscr{B}) \leq r$ and the assertion is proved.

Let $H$ be the open sphere in $C$ about 0 of radius $I(\mathscr{B})$; i.e. $H=\{f:\|f\|<I(\mathscr{B})\}$. Since the norm of each member of $G$ is at least $I(\mathscr{R})$, each member of the combinatorial sum $G+H$ is somewhere positive. If $P$ is the class of non-negative members of $C$ and $Q$ is the cone $\{s(g+h)+t p: s \geq 0, t \geq 0, g \in G, h \in H, p \in P\}$, then no member of $Q$ is everywhere negative. In view of the Hahn-Banach theorem, there is therefore a hyperplane separating $Q$ and the function which is identically -1 ; that is, there is a linear functional $\phi$ such that $\phi(-1)<\phi(f)$ for all $f$ in $Q$. We may suppose that $\phi(-1)=-1$, and because $Q$ is closed under multiplication by positive scalars, we must have $\phi(f)$ $\geq 0$ for $f$ in $Q$. For $e>0$, the function constantly equal to $-I(\mathscr{B})+e$ belongs to $H$, hence $f-I(\mathscr{B})+e \in Q$ for all $f$ in $G$, and therefore $\phi(\mathrm{f}-I(\mathscr{B})+e) \leq 0$. Thus $\phi(f) \geq I(\mathscr{B})-e$ for all positive $e$, and hence $\phi(f) \geq I(\mathscr{B})$ for all $f$ in $G$. Finally, for each $A$ in $\mathscr{A}$ let $m(A)$ be $\phi$ of the characteristic function $K_{A}$ of $A$. Then $m$ is non-negative, finitely additive, $m(X)=1$, and $m(B) \geq I(\mathscr{B})$ if $B \in \mathscr{B}$, because in this case the characteristic function $K_{B}$ belongs to $G$. The theorem is then proved.

3. CoROllary. For each non-void subset $\mathscr{B}$ of $\mathscr{A}$ the intersection number $I(\mathscr{B})$ is the maximum of the numbers inf $\{m(B): B \in \mathscr{B}\}$ for all measures $m$ on $\mathscr{A}$.

4. THEOREM. There is a strictly positive measure on a Boolean algebra $\mathscr{A}$ if and only if $\mathscr{A}-\{0\}$ is the union of a countable number of classes, each of which has a positive intersection number.

Proof. If $\mathscr{A}-\{0\}$ is the union of classes $\mathscr{B}_{n}$ with $I\left(\mathscr{B}_{n}\right)>0$ then, choosing measures $m_{n}$ on $\mathscr{A}$ with inf $\left\{m_{n}(B): B \in \mathscr{B}_{n}\right\}>0$, the $\operatorname{sum} \sum\left\{2^{-n} m_{n}: n\right.$ an integer $\}$ is a strictly positive measure. On the other hand, if $m$ is strictly positive on $\mathscr{A}$ then $\mathscr{A}-\{0\}$ is the union of the classes $\{B: m(B) \geq 1 / n\}$, and each of these classes has a positive intersection number by virtue of Proposition 1 .

We may derive directly from the preceding results a necessary and sufficient condition for the existence of a measure which is "small" on a subclass $\mathscr{C}$ of an algebra $\mathscr{A}$, since $m(A) \leq r$ for each $A$ in $\mathscr{C}$ if 
and only if $m(B) \geq 1-r$ for each member $B$ of the class $\mathscr{B}$ of complements of members of $\mathscr{C}$. This dualization leads to an interesting description of the intersection number of $\mathscr{S}$ in terms of the class $\mathscr{C}$, which we now give.

For each finite sequence $S=\left\langle S_{1}, \cdots, S_{n}\right\rangle$ in $\mathscr{A}$ let $m(S)$, the multiplicity of covering, be the minimum number of times that each point of $X$ is covered by elements of $S$. If $K_{i}$ is the characteristic function of $S_{i}$ then $m(S)=\inf \left\{\sum\left\{K_{i}(x): i=1, \cdots, n\right\}: x \in X\right\}$. Let $n(S)$, as before, be the number $n$ of elements of $S$, and for each nonvoid class $\mathscr{C}$ of $\mathscr{C}$ let the covering number $C(\mathscr{C})$ be the supremum of $m(S) / n(S)$ for all finite sequences $S$ in $\mathscr{C}$. Intuitively, this may be interpreted: $1 / C(\mathscr{C})$ is the outer measure of $X$ obtained by using coverings by elements of $\mathscr{C}$, assigning members of $\mathscr{C}$ measure one, and permitting "multiple" coverings.

The connection between intersection and covering numbers is given by the

5. Proposition. If $\mathscr{P}$ is a non-void subclass of the algebra and $\mathscr{C}$ is the class of complements of members of $\mathscr{P}$, then $I(\mathscr{B})+$ $C(\mathscr{C})=1$.

Proof. Let $S$ be a finite sequence in $\mathscr{A}$. The number $n(S)-i(S)$ can be described: it is the smallest number such that some set of (S) elements of $S$ intersect, and, in terms of the sequence $S^{\prime}=\left\langle S_{1}^{\prime}\right.$, $\left.\cdots, S_{n}{ }^{\prime}\right\rangle$ of complements $S$ of $S=\left\langle S_{1}, \cdots, S_{n}\right\rangle$, the number $n(S)-i(S)$ is the smallest such that the remainder, after omission of some set of $n(S)-i(S)$ elements from $S^{\prime}$, does not cover $X$. In brief, $n(S)-i(S)$ is the multiplicity of covering of $\left\langle S_{1}{ }^{\prime}, \cdots, S_{n}{ }^{\prime}\right\rangle$. We then have : $1-I(\mathscr{B})$ $=1-\inf \{i(S) / n(S): S$ a finite sequence in $\mathscr{P}\}=\sup \{[n(S)-i(S)] / n(S): S$ a finite sequence in $\mathscr{S}\}=\sup \left\{m\left(S^{\prime}\right) / n\left(S^{\prime}\right): S^{\prime}\right.$ a finite sequence in $\mathscr{C}\}=C(\mathscr{C})$.

In view of Corollary 3 , the preceding proposition implies the

6. Corollary. For each non-void subclass $\mathscr{C}$ of $\mathscr{A}$ the covering number $C(\mathscr{C})$ is the minimum of the numbers $\sup \{m(A): A \in \mathscr{C}\}$, where the minimum is taken over all measures $m$ on $\mathscr{A}$.

In view of Theorem 4, we have immediately the

7. Corollary. There is a strictly positive measure on $\mathscr{A}$ if and only if $\mathscr{A}-\{X\}$ is the union of a countable family of subclasses, each of which has covering number less than 1.

Countably additive measures. A Boolean algebra $\mathscr{A}$ is complete if and only if each subclass of $\mathscr{A}$ has a supremum in $\mathscr{A}$ (or equivalently, each subclass has an infimum). We shall denote inf $\{B: B \in \mathscr{B}\}$ by 
$\bigwedge\{B: B \in \mathscr{B}\}$, and $\sup \{B: B \in \mathscr{B}\}$ by $\Lambda\{B: B \in \mathscr{B}\}$. In general these differ from $\cap\{B: B \in \mathscr{B}\}$ and $\bigcup\{B: B \in \mathscr{B}\}$; if the infimum and supremum exist, then $\Lambda\{B: B \in \mathscr{B}\}$ is the interior of $\bigcap\{B: B$ $\in \mathscr{B}\}$, and $\mathrm{V}\{B: B \in \mathscr{B}\}$ is the closure of $\mathrm{U}\{B: B \in \mathscr{B}\}$. In case $\mathscr{A}$ is complete, the interior of each closed subset of $X$ is closed, and the closure of each open subset is open.

In this section it will always be assumed that $\mathscr{A}$ is complete and satisfies the countable chain condition (that is, each disjoint subclass of $\mathscr{A}$ is countable). The set of positive integers will be denoted by $\omega$, and the class of all sequences of positive integers by $\omega^{\omega}$. For $n \in \omega^{\omega}$, and $i \in \omega, n_{i}$ is the $i$ th member of the sequence $n$.

The algebra $\mathscr{A}$ is weakly countably distributive iff for every double sequence $A_{i, j}$ of members of $\mathscr{A}$ such that $A_{i, j+1} \subset A_{i, j}$ for all $i$ and $j$, it is true that $\bigvee\left\{\Lambda\left\{A_{i, j}: j \in \omega\right\}: i \in \omega\right\}=\Lambda\left\{\bigvee\left\{A_{i, n_{i}}: i \in \omega\right\}\right.$ $\left.n \in \omega^{\omega}\right\}$. The topological condition on $X$ which is equivalent to weak countable distributivity is simple and striking. Dixmier has shown [1] that each first category subset of the Stone space of a hyperstonian* algebra is nowhere dense, and the results of Horn and Tarski [2] imply that a hyperstonian algebra is weakly countably distributive. We now show that these two properties are equivalent for complete algebras satisfying the countable chain condition. (I do not believe the following theorem has been published previously, although it has been discovered independently by John Oxtoby.)

8. Theorem. A complete Boolean algebra A which satisfies the countable chain condition is weakly countably distributive if and only if each subset of the Stone $X$ which is of category $I$ is nowhere dense.

Proof. We first note that a subset $A$ of $X$ is nowhere dense if and only if $\Lambda\{B: B \in \mathscr{A}$ and $B \supset A\}=0$. Moreover, because $\mathscr{A}$ satisfies the countable chain condition, the infimum of any subclass $\mathscr{B}$ of $\mathscr{A}$ is identical with the infimum of some countable subclass of $\mathscr{B}$, and hence $A$ is nowhere dense if and only if there is a sequence $\left\{B_{n}\right\}$ of members of $\mathscr{A}$ such that $A \subset B_{n}$ and $\Lambda_{n} B_{n}=0$. Of course, $\left\{B_{n}\right\}$ may be assumed to be monotonically decreasing.

Assuming $\mathscr{A}$ is weakly countably distributive, suppose $C$ is a subset of $X$ which is of the first category. Then $C=\bigcup_{i} A_{i}$, where each $A_{i}$ is nowhere dense, and there are members $B_{i, j}$ of $\mathscr{A}$, monotonically decreasing in $j$, such that $A_{i} \subset \bigcap_{j} B_{i, j}$ and $0=\Lambda_{j} B_{i, j}$ for each $i$. Hence $0=\bigvee_{i} \Lambda_{j} B_{i, j}=\Lambda_{n} \bigvee_{i} B_{i, n_{i}}$. But $\bigvee_{i} B_{i, n_{i}} \supset \bigcup_{i} A_{i}=C$ for each $n$, and it follows that $C$ is nowhere dense.

* That is, the Stone space of a measure algebra. The term "hyperstonian" seems unfortunate. In spite of my affection and admiration for Marshall Stone, I find the notion of a Hyper-Stone downright appalling. 
To prove the converse, suppose that each subset of $X$ which is of the first category is nowhere dense, and that $A_{i, j} \in \mathscr{A}$ and $A_{i, j+j} \subset A_{i, j}$ It is easy to see that $\mathrm{V}_{i} \Lambda_{j} A_{i, i} \subset \Lambda_{n} \bigvee_{i} A_{i, n_{i}}$. Suppose that $B$ is a nonvoid member of $\mathscr{A}$ such that $B \subset \Lambda_{n} \mathrm{~V}_{i} A_{i, n_{i}}$ and $B \cap \mathrm{V}_{i} \Lambda_{j} A_{i, j}$ is void. Since $B \cap \Lambda_{j} A_{i, j}$ is void, $B$ does not intersect the interior of $\bigcap_{j} A_{i, j}$, and hence $B \cap \bigcap_{j} A_{i, j}$ is nowhere dense. Therefore $B \cap \bigcup_{i} \bigcap_{j} A_{i, j}$ is of category one and hence nowhere dense. We may then choose a non-zero member $C$ of $\mathscr{A}$ such that $C \subset B$ and $C \cap \mathrm{U}_{i} \bigcap_{j} A_{i, j}$ is empty. Using compactness, choose $n_{i}$ such that $C \cap A_{i, n_{i}}$ is empty. Then $C \cap \Lambda_{n} \bigvee_{i} A_{i, n_{i}}$ is void, which contradicts the fact $C \subset B$.

Making use of this proposition, we have no difficulty in establishing the

9. THEOREM. Let $\mathscr{A}$ be a complete Boolean algebra with a strictly positive measure $m$. Then there is a strictly positive countably additive measure on $\mathscr{A}$ if and only if $\mathscr{A}$ is weakly countably distributive.

Proof. Horn and Tarski [2] have established the fact that each algebra (not necessarily complete) which has a strictly positive countably additive measure is weakly countably distributive. On the other hand, let $m$ be a strictly positive measure on $\mathscr{A}$ and let $\mathscr{B}$ be the $\sigma$-algebra of subsets of $X$ which is generated by $\mathscr{A}$. Then $m$ has a unique extension $n$ which is a countably additive measure on $\mathscr{B}$. (This may be established by using $m$ and coverings by members of $\mathscr{A}$ to define an outer measure $p$ on $\mathscr{B}$, and showing that the $\sigma$-algebra of $p$ measurable sets contains $\mathscr{A}$; or alternatively, one may use $m$ to define a positive linear functional on the class of continuous linear functions on $X$, and use the Riesz-Kakutani representation theorem for such functionals.) Let $b=\sup \{n(B): B \in \mathscr{B}$ and $B$ nowhere dense $\}$. We assert that this supremum is attained, for if $\left\{B_{i}\right\}$ is a sequence such that $b=\sup \left\{n\left(B_{i}\right): i \in \omega\right\}$ then $D=\bigcup\left\{B_{i}: i \in \omega\right\}$ is also nowhere dense, in view of our hypothesis, and clearly $b=n(D)$. Now define $n_{c}$ by $n_{c}(A)=n(A-D)$. Then, in view of its definition, $n_{c}$ vanishes at every nowhere dense set. Moreover, $n_{c}$ is strictly positive on $\mathscr{A}$, for if $A$ is a non-void member of $\mathscr{A}$ there is a non-void member $B$ of $\mathscr{A}$ such that $B \subset A-D$, and $n_{c}(A) \geq n_{c}(B)=m(B)>0$. Finally, $n_{c}$ is countably additive on $\mathscr{A}$, for if $\left\{A_{i}\right\}$ is a disjoint sequence in $\mathscr{A}$ then the set $E=\mathrm{V}\left\{A_{i}: i \in \omega\right\}-\mathrm{U}\left\{A_{i}: i \in \omega\right\}$ is nowhere dense, hence $n_{c}(E)=0$, and $n_{c}\left(\bigvee\left\{A_{i}: i \in \omega\right\}\right)=n_{c}\left(\bigcup\left\{A_{i}: i \in \omega\right\}\right)=\sum\left\{n_{c}\left(A_{i}\right): i \in \omega\right\}$. The theorem is then proved.

Souslin lines. We first review a few definitions. A linear continum is a non-void set $X$ with a linear ordering $>$ such that $X$ has a first and a last element and such that there are no jumps or gaps. In terms 
of the order topology, these requirements can be stated: $X$ is compact and connected. A linearly ordered set $X$ is of real type iff there is an order isomorphism of $X$ onto a subset of the class of real numbers. The interval algebra of a linearly ordered set is the Boolean algebra generated by the half-open intervals $(a: b]=\{x: a<x \leq b\}$, where $a$ and $b$ are arbitrary points of $X$. It is well known (and easy to prove) that a linear continuum $X$ is of real type iff the order topology is separable (that is, there is a countable dense subset of $X$ ), and this is the case iff there is a strictly positive measure on the interval algebra of $X$. If the interval algebra satisfies the countable chain condition, then $X$ is said to satisfy the countable interval condition.

We shall call a linear continuum $X$ a Souslin line iff $X$ satisfies the countable interval condition, and no non-void interval in $X$ is of real type. We now show that, if the Souslin hypothesis fails, then there exists a Souslin line.

10. Proposition. Let $X$ be a linear continuum which satisfies the countable interval condition but is not of real type. If $R$ is the relation $\{(y, y): x=y$ or the interval $[y, x]$ is of real type or the interval $[x: y]$ is of real type\}, then the quotient $X / R$ is, with the induced order, a Souslin line.

Proof. As a preliminary we show that if $\mathscr{T}$ is a family of intervals in $X$ which is linearly ordered by inclusion, and if each member of $\mathscr{T}$ is of real type, then $\bigcup\{T: T \in \mathscr{T}\}$ is also of real type. If $\mathscr{T}$ is countable this result follows from the fact that a countable union of separable subsets of $X$ is itself separable. But $\mathscr{T}$ may always be assumed to be countable for: we may by transfinite induction choose a subfamily $\left\{T_{a}\right\}$ of $\mathscr{T}$ which is well ordered by $\subset$ and covers $\bigcup\{T: T$ $\in \mathscr{T}\}$, and such a well ordered family must be countable since otherwise the class of sets of the form $T_{a+1}-T_{a}$ yields an uncountable disjoint family of intervals.

It follows easily from the above that an equivalence class modulo the relation $R$ is either a closed interval or consists of a single point, and that the family $\mathscr{T}$ of such intervals must be countable and disjoint. The quotient map $Q$ of $X$ onto $X / R$ is then continuous relative to the order topologies for $X$ and $X / R$, and $X / R$ is therefore compact and connected. Finally, suppose that $I$ is a separable interval in $X / R$, that $A$ is a countable dense subset of $I$, and that $B$ is a countable dense subset of $\bigcup\{F: F \in \mathscr{T})$. Let $C$ be a countable subset of $X$ such that $B \subset C$ and $C$ intersects $Q^{-1}[x]$ for each $x$ in $A$. We assert that the closure $C^{-}$of $C$ contains $Q^{-1}[I]$, for : if $J$ is an open interval disjoint from $C^{-}$then $J$ is disjoint from $\bigcup\{F: F \in \mathscr{T}\}$, hence $Q$ is a homeomorphism on $J$, whence $Q[J]$ is disjoint from $A$, and therefore $Q[J]$ 
is disjoint from $I$. Thus $Q^{-1}[I]$ is separable, and this, in view of the definition of $R$, contradicts the fact that $I$ is a proper interval. Consequently $X / R$ can contain no separable interval, and is therefore a Souslin line.

It is now easy to show that a Souslin line has very curious properties. Recall that a regular open set is an open set which is the interior of its closure. For a compact Hausdorff space (or for any space which is of the second category at each of its points) the Boolean algebra of regular open sets is naturally isomorphic to the algebra of Borel sets modulo the ideal of Borel sets of the first category, for: the class of all Borel sets $A$ such that the Boolean sum of $A$ and some first category set $B$ is regular and open is easily seen to contain the Borel algebra, and no non-void regular open set is of category $I$.

11. Lemma. Let $X$ be a Souslin line. Then a subset $A$ of $X$ is separable if and only if it is nowhere dense, and this is the case if and only if the set $A$ is of the first category.

Proof. A separable subset of $X$ is nowhere dense in view of the definition of a Souslin line; conversely, if $A$ is nowhere dense the set $E$ of endpoints of intervals complementary to $A^{-}$is countable, and choosing a member of $A$ between each pair of points of $E$ whenever possible yields a countable dense subset of $A$. Finally, each nowhere dense set is of the first category, and the countable union of sets which are nowhere dense, hence separable, is separable, and hence nowhere dense.

12. THEOREM. The algebra $\mathscr{A}$ of regular open subsets of a Souslin line $X$ has the properties:

(i) each disjoint subfamily of $\mathscr{A}$ is countable,

(ii) the algebra $\mathscr{A}$ is complete,

(iii) the algebra $\mathscr{A}$ is weakly countably distributive,

(iv) there is no strictly positive measure on $\mathscr{A}$, and in fact

(v) if $m$ is any measure on $\mathscr{A}$ then there is a countable subfamily $\mathscr{B}$ of $\mathscr{A}$ such that $m(B)=0$ for $B$ in $\mathscr{B}$ and $\mathrm{V}\{B: B \in \mathscr{B}\}=X$.

Proof. The assertion (i) is clear, and (ii) follows from (i) together with the fact that $\mathscr{A}$ is countably complete. To show that $\mathscr{A}$ is weakly countably distributive let us consider $\mathscr{A}$ as the algebra of Borel subsets of $X$ modulo sets of the first category, and suppose that $A_{i, j}$ is a double sequence of Borel sets such that $A_{i, j} \supset A_{i, j+1}$ for all $i$ and $j$. We may suppose that each $A_{i, j}$ is closed. It is always the case that $B=\Lambda\{\mathrm{V}$ $\left.\left\{A_{i, n_{i}}: i \in \omega\right\}: n \in \omega^{\omega}\right\} \supset \mathrm{V}\left\{\Lambda\left\{A_{i, j}: j \in \omega\right\}: i \in \omega\right\}=C$. Let us suppose that $I$ is a non-void open interval whose intersection with $C$ is of 
the first category, (and hence nowhere dense). Then $I$ contains a nonvoid open interval $J$ whose closure is disjoint from $C$. Via compactness, choose for each $i$ an integer $n_{i}$ such that $A_{i, n_{i}}$ fails to intersect $J$. Then $B$, in view of its definition, is disjoint from $J$, and we have showed that $B \cap(X-C)$ is nowhere dense. Thus $B$ is congruent to $C$ modulo the class of sets of the first category, and $\mathscr{A}$ is weakly countable distributive.

If there were a strictly positive measure on $\mathscr{A}$ then the map carrying each point $x$ of $X$ into the measure of the interval $[a, x]$, where $a$ is the first point of $X$, would be an order isomorphism of $X$ into a set of real numbers. Thus (iv) is proved, and assertion (v) follows from a simple argument based on the fact that every closed interval in $X$ is itself a Souslin line.

REMARK. Part (iv) above may easily be strengthened; for example: there is clearly no strictly monotonic real valued function on $\mathscr{A}$.

It is not difficult to give a precise equivalence to the Souslin hypothesis in terms of properties of Boolean algebras. Let us call a maximal chain in a Boolean algebra (that is, a maximal linearly ordered subclass) a segment. A Boolean algebra is atomless if and only if each non-zero element is the sum of two disjoint non-zero elements.

13. THEOREM. The following statements are equivalent:

(i) (the Souslin hypothesis) each linear continuum which satisfies the countable interval condition is of real type,

(ii) each segment in an atomless Boolean algebra which satisfies the countable chain condition is of real type, and

(iii) if $\mathscr{A}$ is the algebra of regular open subsets of a linear continuum, and if $\mathscr{A}$ is complete, atomless, weakly countably distributive, and satisfies the countable chain condition, then each segment in $\mathscr{A}$ is of real type.

Proof. We first show that (i) implies (ii). If $\mathscr{S}$ is a segment in atomless Boolean algebra $\mathscr{A}$ which satisfies the countable chain condition, then it is easily seen that there is no uncountable disjoint family of intervals in $\mathscr{S}$. Moreover, since $\mathscr{A}$ is atomless, there are no gaps in $\mathscr{S}$ (that is, between any two distinct members of $\mathscr{S}$ there is a third member which is distinct from both). It follows that the (order) completion of $\mathscr{S}$ is a linear continuum satisfying the countable interval condition, and, assuming the Souslin hypothesis, is of real type. Thus $\mathscr{S}$ is of real type.

Clearly (ii) implies (iii). That (iii) implies (i) is an immediate consequence of the properties of a Souslin line, and the fact (Proposition 10) that a Souslin line exists if the Souslin hypothesis fails. 
Dominated measures. Maharam has showed [3] that if a Boolean algebra $\mathscr{A}$ satisfies certain conditions then there is a continuous outer measure on $\mathscr{A}$; that is, there is a non-negative real valued function $p$ such that if $A=\bigvee\left\{A_{n}: n \in \omega\right\}$ then $p(A) \leq \sum\left\{p\left(A_{n}: n \in \omega\right\}\right.$, and, if $\left\{A_{n}\right)$ is a monotonically decreasing sequence with $0=\Lambda\left\{A_{n}: n \in \omega\right\}$ then $p\left(A_{n}\right)$ converges to zero. It seems possible that this result might be strengthened by showing that each outer measure dominates a measure. This leads to the general problem of Hahn-Banach type: if $\mathscr{B}$ is a subalgebra of $\mathscr{A}$ and if $m$ is a measure on $\mathscr{B}$ which is dominated there by a non-negative function $p$ such that $p(A)+p(B) \geq p(A \cup B)$, is it then possible to find a measure on $\mathscr{A}$ which is an extension of $m$ and is everywhere dominated by $p$ ? The extension theorem just proposed is false, even for finite algebras. However, a similar result can be established if the premises concerning the function $p$ are strengthened, and, although the result fails to apply to Maharam's theorem, it appears to be of some interest in itself.

14. Theorem. Let $\mathscr{A}$ be a Boolean algebra, let $p$ be a non-negative monotonic real valued function on $\mathscr{A}$ such that $p(A)+p(B) \geq p(A \cup B)$ $+p(A \cap B)$ for all members $A$ and $B$ of $\mathscr{A}$, and let $m$ be a measure on a subalgebra $\mathscr{B}$ of $\mathscr{A}$ such that $m(B) \leq p(B)$ for $B$ in $\mathscr{B}$. Then there is a measure $n$ on $\mathscr{A}$, which is an extension of $m$, such that $n(A) \leq p(A)$ for all $A$ in $\mathscr{A}$.

Proof. The proof is first reduced, by means of a compactness argument, to the case of a finite Boolean algebra. For each pair $\mathscr{C}$ and $\mathscr{D}$ of finite subalgebras of $\mathscr{A}$ such that $X \in \mathscr{C} \subset \mathscr{S}$ and $\mathscr{C} \subset$ $\mathscr{O}$ let $Q(\mathscr{C}, \mathscr{D})$ be the set of all non-negative functions $q$ on $\mathscr{Z}$ which satisfy the requirements: $q$ is finitely additive on $\mathscr{D}, q(C)=m(C)$ for $C$ in $\mathscr{C}$, and $q(A) \leq p(A)$ for $A$ in $\mathscr{A}$. The class $Q(\mathscr{C}, \mathscr{D})$ is, by virtue of the Tychonoff product theorem, compact relative to the topology of pointwise convergence on $\mathscr{A}$, and $Q\left(\mathscr{C}^{\prime}, \mathscr{D}\right) \supset Q(\mathscr{C}, \mathscr{D})$ if $\mathscr{C}^{\prime} \subset$ $\mathscr{C}$ and $\mathscr{D}^{\prime} \subset \mathscr{D}$. Proof of the theorem is equivalent to showing that the intersection of the classes $Q(\mathscr{C}, \mathscr{O})$, for all $\mathscr{C}$ and $\mathscr{D}$, is non-void, and in view of compactness it is sufficient to show that each class $Q(\mathscr{C}, \mathscr{D})$ is non-void.

The problem is then reduced to that of extending $m$ from a subalgebra $\mathscr{C}$ to a finite containing subalgebra $\mathscr{D}$, and we may assume that $\mathscr{D}$ is a minimal algebra properly containing $\mathscr{C}$. In this case, using the known structure of finite Boolean algebras, $\mathscr{C}$ is generated by a finite class $C, C_{1}, \cdots, C_{n}$ of disjoint non-void sets, and $\mathscr{D}$ is generated by $D, D^{\prime}, C_{1}, \cdots, C_{n}$, where $D \cap D^{\prime}$ is void and $D \cup D^{\prime}=C$. The extension of $m$ requires the choice of a number $m(D)$ such that the following inequalities are satisfied: 
$m(D)+m(A) \leq p(D \cup A)$ for all $A$ in $\mathscr{C}$ with $A \cap C$ void, and $m\left(D \cup D^{\prime}\right)-m(D)+m(B) \leq p\left(D^{\prime} \cap B\right)$ for all $B$ in $\mathscr{C}$ with $B \cup C$ void. Thus extension is possible if and only if $m(D)$ can be chosen so that $m(C)+m(B)-p\left(D^{\prime} \cup B\right) \leq m(D) \leq p(D \cup A)-m(A)$ for all members $A$ and $B$ of $\mathscr{C}$ which are disjoint from $C$, and this inequality can be attained if the left hand member never exceeds the right hand for all such choices of $A$ and $B$. Rewriting, the proof reduces to establishing that $m(C)+m(A)+m(B) \leq p(D \cup A)+p\left(D^{\prime} \cup B\right)$ for all members $A$ and $B$ of $\mathscr{C}$ which are disjoint from $C$. But

$$
\begin{aligned}
& m(C)+m(A)+m(B)=m(C \cup A \cup B)+m(A \cap B) \\
& \leq p(C \cup A \cup B)+p(A \cap B) \leq p(D \cup A)+p\left(D^{\prime} \cap B\right),
\end{aligned}
$$

the last inequality being derived from the assumption on $p$ as applied to the sets $D \cup A$ and $D^{\prime} \cup B$. Thus the extension of $m$ is always possible, and the theorem is proved.

There is a dual to the preceding theorem which may be obtained as follows: Suppose $m$ is a measure on the subalgebra $\mathscr{B}$ and that $m$ dominates a non-negative function $p$ such that $p(A)+p(B) \leq p(A \cup B)$ $+p(A \cap B)$ for all $A$ and $B$ in $\mathscr{A}$. Then, setting $q(A)=m(X)-p(X-A)$, it is easily verified that $m$ and $q$ satisfy the conditions of the preceding theorem. There is therefore an extension $n$ of $m$ which is everywhere dominated by $q$, and it follows that $n$ dominates $p$. Hence:

15. Corollary. Let $\mathscr{A}$ be a Boolean algebra, let $p$ be a non-negative monotonic real valued function on $\mathscr{A}$ such that $p(A)+p(B) \leq p(A \cup B)$ $+p(A \cap B)$ for all $A$ and $B$ in $\mathscr{C}$, and let $m$ be a measure on $a$ subalgebra $\mathscr{S}$ of $\mathscr{A}$ such that $m(B) \geq p(B)$ for $B$ in $\mathscr{B}$. Then there $i s$ a measure $n$ on $\mathscr{X}$, which is an extension of $m$, such that $n(A) \geq$ $p(A)$ for all $A$ in $\mathscr{A}$.

\section{ADDENDUM}

Since writing this paper I have received the following communications. From Professor A. Horn (June 18, 1959) :

A. "... Lemma 11 - this property is actually characteristic of Souslin lines: A linear countinuum in which the separable subsets coincide with the nowhere dense subsets is a Souslin line, and conversely. This is true because if we have an uncountable disjoint family of intervals, then a set formed by choosing one point from each interval is nowhere dense and non separable. Thus we have a new and intersting formulation of Souslin's problem ...."

B. $[\cdots .$. Incidentally, it is interesting that Theorem 14 is not valid (even for monotonic $p$ ), if $\mathscr{B}$ is not a subalgebra and $m$ is merely a partial measure (in the sense of [2]) on $\mathscr{B} \cdots$ ] 
From Professor Roman Sikorski :

March 25, 1959.

A. (1) The proof of Theorem 2 can be simplified. Theorem 2 is a particular case of the following general theorem:

$\left.{ }^{*}\right)$ Let $X$ be a partially ordered Banach space such that $0 \leq x \leq y$ implies $|x| \leq|y|$. For every convex set $S$ of non-negative elements there exists a functional $f \geq 0^{1}$ such that $|f|=1$ and $\inf _{x \in S} f(x)=\inf _{x \in S}|x|$.

Theorem $(*)$ follows immediately from a general theorem on the existence of a functional satisfying a given set of inequalities. This general theorem is due to Mazur and Orlicz (Studia Math. 13, (1953), 137-179). A simple proof of Mazur-Orlicz's theorem was given by me (ibidem, p. 180) and by Ptak (also in Studia Math.).

(2) Your Theorem 9 can be proved simply without using Stone spaces. In fact, suppose that $m$ is a finite measure on a Boolean algbra $\mathscr{B}$. The formula

$$
m^{\prime}(A)=\inf \left(m\left(A_{1}\right)+m\left(A_{2}\right)+\cdots\right) \quad \text { for } A \in \mathscr{B}
$$

(where inf is extended over all disjoint decompostions $A=A_{1}+A_{2}$ $+\cdots$ ) defines a $\sigma$-measure on $\mathscr{B}$ (viz. $m^{\prime}$ is the greatest $\sigma$ -measure $\leq m$ ). It is easy to verify that if $m$ is strictly positive and $B$ is weakly $\sigma$-distributive, then $m^{\prime}$ is strictly positive.

The remark (2) is due to Professor Ryll-Nardzewski.

April 3, 1959

B. I would like to inform you that Professor Ryll-Nardzewski has found the following analogue of your Theorem 4 for $\sigma$-measures:

$\left({ }^{*}\right)$ There exists a strictly positive $\sigma$-measure $\mu$ on a Boolean algebra $\mathscr{B}$ if and only if $\mathscr{B}-(0)$ is the union of a sequence $\left\{\mathscr{B}_{n}\right\}$ such that, for every $n$,

(1) the intersection number $I\left(\mathscr{B}_{n}\right)$ is positive;

(2) if $A_{m} \subset A_{m+1}(m=1,2, \cdots)$ and $A_{1}+A_{2}+\cdots \in \mathscr{S}_{n}$, then there exists an $m$ such that $A_{m} \in \mathscr{B}_{n}$.

Necessity. Take as $\mathscr{B}_{n}$ the class of all $A \in \mathscr{B}$ such that $\mu(A)>1 / n$.

Sufficiency. There exists a measure $\mu_{n}^{\prime}$ such that $\mu_{n}^{\prime}(A) \geq$ $I\left(\mathscr{B}_{n}\right)$ for every $A \in \mathscr{B}_{n}$. Let

$$
\mu_{n}(A)=\inf \lim _{m} \mu_{n}{ }^{\prime}\left(A_{m}\right)
$$

where inf is extended over all sequences $A_{m} \in \mathscr{B}$ such that $A=A_{1}+A_{2}+\cdots$ and $A_{m} \subset A_{m+1}$. By definition, $\mu_{n}$ is a $\sigma$-measure and $\mu_{n}(A) \geq I\left(\mathscr{B}_{n}\right)$ for $A \in \mathscr{B}_{n}$ on account of (2). The $\sigma$-measure

1 i.e. such that $f(x) \geq 0$ for $x \geq 0$. 


$$
\mu(A)=\frac{1}{2} \mu_{1}(A)+\frac{1}{2^{2}} \mu_{2}(A)+\cdots
$$

is strictly positive on $B$.

\section{REFERENCES}

1. J. Dixmier, Sur certains espaces considérés par M. H. Stone, Summa Brasil. Math. 2 (1951), 151-182.

2. A. Horn and A. Tarski, Measures in Boolean algebras, Trans. Amer. Math. Soc. 64 (1948), 467-497.

3. D. Maharam, An algebraic characterization of measure algebras, Annals of Math., 48 (1947), 154-167.

UNIVERSITY OF CALIFORNIA

CAMBRIDGE UNIVERSITY 



\section{PACIFIC JOURNAL OF MATHEMATICS}

EDITORS

David Gilbarg

Stanford University

Stanford, California

R. A. Beaumont

University of Washington

Seattle 5 , Washington
A. L. Whiteman

University of Southern California

Los Angeles 7, California

L. J. PAIGE

University of California

Los Angeles 24, California

\section{ASSOCIATE EDITORS}

\author{
E. F. BECKENBACH \\ C. E. BURGESS \\ E. HEWITT \\ A. HORN
}

\author{
V. GANAPATHY IYER \\ R. D. JAMES \\ M. S. KNEBELMAN \\ L. NACHBIN
}
I. NIVEN
E. G. STRAUS
T. G. OSTROM
G. SZEKERES
H. L. ROYDEN
F. WOLF
M. M. SCHIFFER
K. YOSIDA

\section{SUPPORTING INSTITUTIONS}

\author{
UNIVERSITY OF BRITISH COLUMBIA \\ CALIFORNIA INSTITUTE OF TECHNOLOGY \\ UNIVERSITY OF CALIFORNIA \\ MONTANA STATE UNIVERSITY \\ UNIVERSITY OF NEVADA \\ OREGON STATE COLLEGE \\ UNIVERSITY OF OREGON \\ OSAKA UNIVERSITY \\ UNIVERSITY OF SOUTHERN CALIFORNIA
}

\author{
STANFORD UNIVERSITY \\ UNIVERSITY OF TOKYO \\ UNIVERSITY OF UTAH \\ WASHINGTON STATE COLLEGE \\ UNIVERSITY OF WASHINGTON \\ $* \quad * \quad *$ \\ AMERICAN MATHEMATICAL SOCIETY \\ CALIFORNIA RESEARCH CORPORATION \\ HUGHES AIRCRAFT COMPANY \\ SPACE TECHNOLOGY LABORATORIES
}

Mathematical papers intended for publication in the Pacific Journal of Mathematics should be typewritten (double spaced), and the author should keep a complete copy. Manuscripts may be sent to any one of the four editors. All other communications to the editors should be addressed to the managing editor, L. J. Paige at the University of California, Los Angeles 24, California.

50 reprints per author of each article are furnished free of charge; additional copies may be obtained at cost in multiples of 50 .

The Pacific Journal of Mathematics is published quarterly, in March, June, September, and December. The price per volume (4 numbers) is $\$ 12.00$; single issues, $\$ 3.50$. Back numbers are available. Special price to individual faculty members of supporting institutions and to individual members of the American Mathematical Society: $\$ 4.00$ per volume; single issues, $\$ 1.25$.

Subscriptions, orders for back numbers, and changes of address should be sent to Pacific Journal of Mathematics, 2120 Oxford Street, Berkeley 4, California.

Printed at Kokusai Bunken Insatsusha (International Academic Printing Co., Ltd.), No. 6, 2-chome, Fujimi-cho, Chiyoda-ku, Tokyo, Japan.

PUBLISHED BY PACIFIC JOURNAL OF MATHEMATICS, A NON-PROFIT CORPORATION

The Supporting Institutions listed above contribute to the cost of publication of this Journal, but they are not owners or publishers and have no responsibility for its content or policies. 


\section{Pacific Journal of Mathematics}

\section{Vol. 9, No. 4 \\ August, 1959}

Frank Herbert Brownell, III, A note on Kato's uniqueness criterion for

Schrödinger operator self-adjoint extensions ............... 953

Edmond Darrell Cashwell and C. J. Everett, The ring of number-theoretic

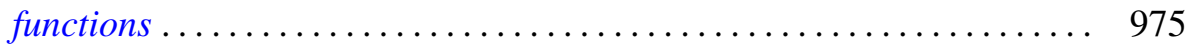

Heinz Otto Cordes, On continuation of boundary values for partial

differential operators ............................. 987

Philip C. Curtis, Jr., n-parameter families and best approximation . . . . . . 1013

Uri Fixman, Problems in spectral operators . . . . . . . . . . . . . . . 1029

I. S. Gál, Uniformizable spaces with a unique structure .............. 1053

John Mitchell Gary, Higher dimensional cyclic elements ............ 1061

Richard P. Gosselin, On Diophantine approximation and trigonometric

polynomials ..................................... 1071

Gilbert Helmberg, Generating sets of elements in compact groups ........ 1083

Daniel R. Hughes and John Griggs Thompson, The H-problem and the

structure of $H$-groups .................................. 1097

James Patrick Jans, Projective injective modules ................. 1103

Samuel Karlin and James L. McGregor, Coincidence properties of birth and

death processes ..................................... 1109

Samuel Karlin and James L. McGregor, Coincidence probabilities ........ 1141

J. L. Kelley, Measures on Boolean algebras ................... 1165

John G. Kemeny, Generalized random variables ................... 1179

Donald G. Malm, Concerning the cohomology ring of a sphere bundle ... . . 1191

Marvin David Marcus and Benjamin Nelson Moyls, Transformations on

tensor product spaces .................................. 1215

Charles Alan McCarthy, The nilpotent part of a spectral operator ........ 1223

Kotaro Oikawa, On a criterion for the weakness of an ideal boundary

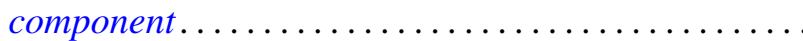

Barrett O'Neill, An algebraic criterion for immersion . . ............... 1239

Murray Harold Protter, Vibration of a nonhomogeneous membrane ... . . . . 1249

Victor Lenard Shapiro, Intrinsic operators in three-space . . . . . . . . . . . 1257

Morgan Ward, Tests for primality based on Sylvester's cyclotomic

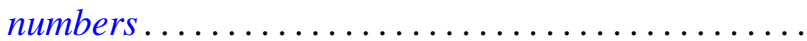

L. E. Ward, A fixed point theorem for chained spaces ....

Alfred B. Willcox, Šilov type $C$ algebras over a connected locally compact

abelian group..................................... 1279

Jacob Feldman, Correction to "Equivalence and perpendicularity of

Gaussian processes" ........................ 